\title{
Heparin-Induced Thrombocytopenia Complicating Thrombotic Thrombocytopenic Purpura
}

\author{
Galila F. Zaher, MD, FRCPath and Maha A. Badawi, MBBS, FRCPC, DRCPSC \\ Department of Hematology, Faculty of Medicine \\ King Abdulaziz University, Jeddah, Saudi Arabia
}

\section{Correspondence}

Dr. Galila F. Zaher

P.O. Box 80215, Jeddah 21589, Saudi Arabia

e.M: zgalila@yahoo.co.uk

Submission: 25 Oct. 2016

Accepted: 13 Dec. 2016

\section{Citation}

Zaher GF, Badawi MA. Heparin-liduced thrombocytopenia complicating thrombotic thrombocytopenic purpura. JKAU Med Sci 2017; 24 (1): 43-48. DOI: 10.4197/Med. 23.4.5

\begin{abstract}
Since the introduction of therapeutic plasma exchange for the management of thrombotic thrombocytopenic purpura, the prognosis of this disease improved significantly. Some patients suffer from refractory disease and adjunctive therapy needs to be considered. In addition, alternative explanations for thrombocytopenia may be present. In this report we discuss a patient who presented with typical findings of thrombotic thrombocytopenic purpura and responded initially to therapeutic plasma exchange and steroids. Shortly afterwards, his platelet count deteriorated and he was found to have acute pulmonary embolism. Prior to the pulmonary embolism, the patient had received venous thromboembolism prophylaxis in the form of low molecular weight heparin and had a history of previous exposure to unfractionated heparin. Testing for heparin-induced thrombocytopenia antibodies was positiveand the patient was started on an alternative anticoagulant. Despite these early interventions the patient did not survive. It is essential that physicians be aware of such possible associations to request appropriate investigations and start appropriate management, accordingly.
\end{abstract}

\section{Keywords}

Thrombotic thrombocytopenic purpura; Heparin induced thrombocytopenia

\section{Introduction}

hrombotic thrombocytopenic purpura (TTP) is a particular form of thrombotic microangiopathies typically characterized by microangiopathic hemolytic anemia, profound thrombocytopenia, fever, neurological complications and renal impairment. Deficiency of a disintegrin and metalloprotease with thrombospondin repeat, member 13 (ADAMTS13), which has a von Willebrand factor-cleaving activity, has been associated with TTP ${ }^{[1]}$.
Since the introduction of therapeutic plasma exchange (TPE) as the main treatment in TTP, fatality rate dropped significantly from $80-90 \%$ to $10-20 \%{ }^{[2]}$. However, some patients do not respond adequately to TPE and require additional measures, such as steroids, rituximab, bortezomib, among other measures ${ }^{[3]}$. It is common practice to use some of these agents before any evidence that disease is refractory. Although refractory disease should be considered in patients who fail to sustain response to TPE, it is essential to contemplate alternative explanations for thrombocytopenia. 
In this report, we describe the case of a patient who presented initially with classic TTP but was later diagnosed as heparin-induced thrombocytopenia and thrombosis (HITT). Co-existence of these conditions have been reported only twice ${ }^{[4,5]}$, and it is extremely important that physicians managing such patients be aware of this phenomenon, as it affects treatment decisions dramatically.

\section{Case Report}

A 59-year-old male patient was brought to the emergency department with history of confusion and deterioration in the level of conciseness for one day. The patient was known to have ischemic heart disease and had a stent insertion through percutaneous coronary intervention three years earlier. He was on aspirin, clopidogrel, atorvastatin, bisoprolol, and captopril. Physical examination revealed fever, pallor, and confusion. The blood film showed significant schistocytosis, and serology for HBV, HCV and HIV was negative. Non-enhanced CT brain was normal.

Based on the presence of microangiopathic hemolytic anemia, severe thrombocytopenia, fever, neurological changes, and renal injury, the patient was diagnosed as TTP, however testing for ADAMTS13 level was not available. Screening tests looking for an underlying malignancy and autoimmune disorders were negative. The patient underwent urgent insertion of an apheresis catheter and was started on TPE using Spectra Optia Apheresis System (Terumo BCT, Inc., Lakewood, CO USA). The patient's blood group was $A B$ Rh negative and fresh frozen plasma was used as replacement fluid and citrate was used as an anticoagulant. Daily TPE were performed on days 1-6, $8,10-13$, and 15-18, targeting removal of at least 1 plasma volume. The patient's level of consciousness improved dramatically after the second session of TPE. Considering the patient's blood group, it was not feasible to supply adequate amounts of FFP in the volumes that were required; therefore, solvent detergent plasma of $A B$ group was purchased to maintain an adequate supply of plasma.

On Day 3, the patient developed a tonic clonic seizure that was managed with diazepam and necessitated intubation. Repeat unenhanced CT scan of the head excluded the presence of hemorrhage. He was started on steroids and levetiracetam and TPE was continued. The patient's level of consciousness improved as well as his renal functions, hemolytic parameters and the platelet count (Fig. 1). The patient was extubated on Day 6. As platelet count was above

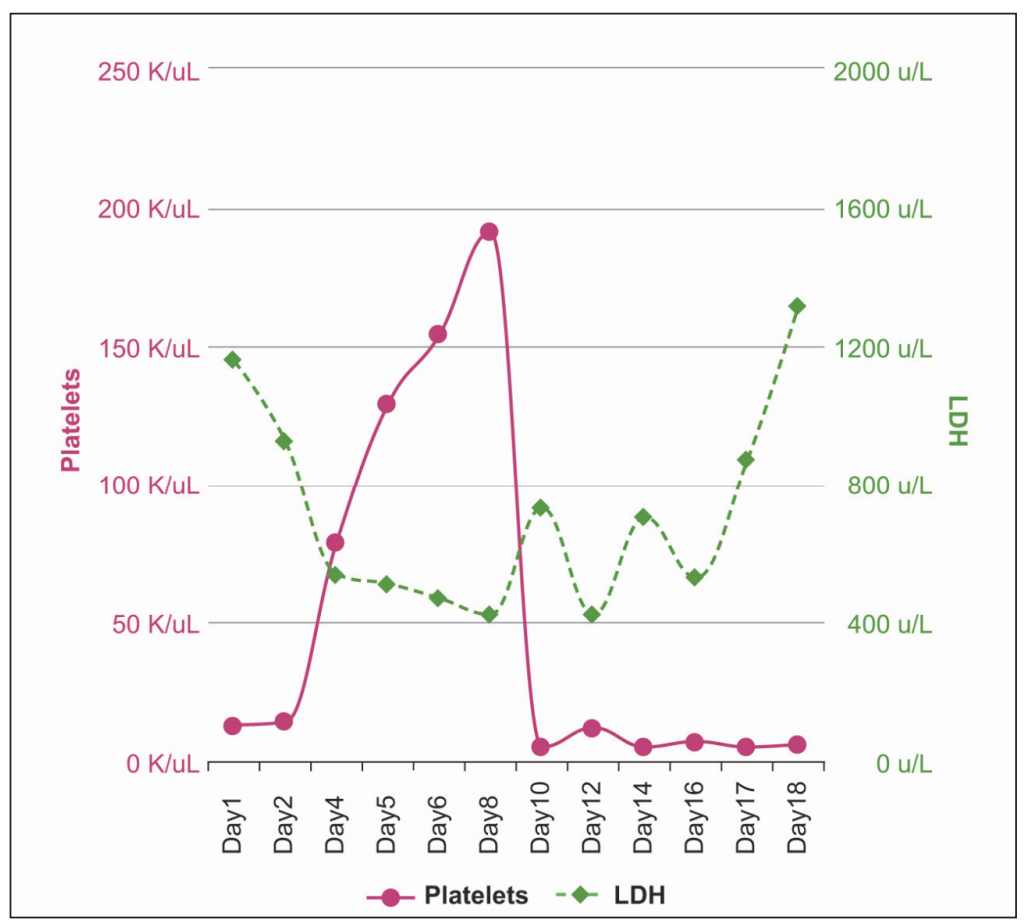

Figure 1. Results of platelets and lactate dehydrogenase (LDH) throughout hospitalization. 
100 by Day 5, prophylactic low molecular weight heparin (LMWH) (enoxaparin $40 \mathrm{mg}$ subcutaneously daily) was started.

However, the improvement was short lived and the patient dropped his platelets count again by Day 10 , so rituximab was started as a $2^{\text {nd }}$ line management of TTP.

Rituximab was given at a dose of $375 \mathrm{mg} / \mathrm{m}^{2} /$ week. Two doses were administered during his hospitalization (Days 10 and 17). On Day 14, the patient was noted to be short of breath, tachypneic and had an altered level of consciousness. Investigations showed that hemoglobin was $11.3 \mathrm{~g} / \mathrm{dL}$, platelets were $5 \times 10^{9} / \mathrm{L}$, creatinine was $101 \mathrm{mmol} / \mathrm{L}$, and LDH was $704 \mathrm{u} / \mathrm{L}$. Coagulation profile continued to be normal. Differential diagnosis of his acute deterioration included refractory TTP, drug-induced thrombocytopenia (including HIT), sepsis, or disseminated intravascular coagulation (DIC). Normal coagulation profile excluded DIC and brain CT with contrast excluded acute insult. However, CT pulmonary angiogram revealed pulmonary emboli in the right lower lobar and left lower segmental branches of the pulmonary vasculature. Bilateral lower limb Doppler ultrasound were negative for an embolic source. As HITT was highly suspected, the patient was evaluated using the 4T score. Thrombocytopenia, pulmonary embolism, timing in relation to heparin exposure (as the patient had a history of previous exposure to UFH during cardiac surgery in addition to current use of LMWH for VTE prophylaxis and heparin flushes in the apheresis catheter), and the presence of a possible alternative explanation for thrombocytopenia in our patient added to a total score of 5 .

Low molecular weight heparin was immediately discontinued and samples were sent for HITT particle gel immunoassay. Owing to unavailability of argatroban and fondaparinux, the patient was started on rivaroxaban $15 \mathrm{mg}$ every 12 hours. Heparininduced thrombocytopenia assay was positive, but the patient's clinical status and hematological parameters did not improve. The patient was put on mechanical ventilation but unfortunately, he arrested on Day 18 and resuscitation attempts were unsuccessful.

\section{Discussion}

Thrombotic thrombocytopenic purpura is a well characterized syndrome resulting in multiorgan dysfunction. The classic pentad includes thrombocytopenia, microangiopathic hemolytic anemia, neurologic symptoms, renal failure and fever ${ }^{[6]}$. Despite introduction of successful interventions for management of TTP, a number of features were recently identified as associated with increased risk of mortality ${ }^{[3]}$. Risk stratification can identify patients who could benefit from early adjunctive therapy. Thrombotic thrombocytopenic purpura requires a rapid diagnosis and an adapted management in emergency. Daily sessions of TPE until durable platelet count recovery remain the basis of management of acquired TTP ${ }^{[1]}$. Based on published evidence, TPE is classified as category I in American Society for Aphaeresis Guidelines, being an effective modality as first line treatment in TTP ${ }^{[2]}$. Anti-CD20 monoclonal antibody (rituximab) has been increasingly used in patients with a suboptimal response to standard treatment or in case of an exacerbation of the disease despite intensive TPE ${ }^{[7]}$. Upon recovery of the platelets count it is generally considered appropriate to start TTP patients on VTE prophylaxis. As illustrated in this case, exposure to heparin puts patients at risk of developing HITT $^{[7]}$. Catastrophic presentation of TTP rarely can involve large vessel infarction ${ }^{[6]}$. Furthermore, catastrophic antiphospholipid syndrome (CAPS) has clinical and laboratory features. The unique characteristics of CAPS are: Rapid onset of thromboses resulting in multi-organ failure; common association with other thrombotic microangiopathies; evidence of systemic inflammatory response syndrome and relatively high mortality ${ }^{[8]}$. The diagnosis requires a careful assessment in patients who may have other explanations for multiple organ thromboses ${ }^{[9]}$. In CAPS multiple organ thromboses is usually accompanied by microthrombi and hematologic manifestations. The clinical manifestations of CAPS may evolve gradually overlapping with other thrombotic microangiopathies, requiring a high index of clinical suspicion. Thetreatment of CAPS should be initiated urgently even without the confirmatory antiphospholipid (APL) antibody tests to prevent high fatality of the disease ${ }^{[8]}$. Some antiphospholipid-positive patients with multiple organ thromboses and/or thrombotic microangiopathies exist who do not fulfill the diagnostic criteria. However, previous antiphospholipid syndrome diagnosis and/or persistent clinically significant APL positivity is of great importance for the CAPS diagnosis. The diagnostic challenges arise where almost half of the patients who develop CAPS do not have a history of APL positivity ${ }^{[10]}$.

However, this diagnosis was less entertained as the patient had negative lupus anticoagulant, negative anti 
cardio lipin antibodies (both $\lg \mathrm{G}$ and $\lg \mathrm{M}$ ) and negative B2 glycoproteins antibodies (both lgG and lgM).

The previous history of heparin exposure in our case suggested (HITT). Four components are thought to be essential for development of HITT: heparin, platelet factor 4 (PF4), IgG antibodies against the HepPF4 complex, and platelet FcyRlla ${ }^{[11]}$. The syndrome is characterized by a thrombocytopenia and venous or arterial thrombosis after 5-10 days of heparin treatment. An earlier presentation could occur in patients that were previously exposed to heparin. The diagnosis rests on a clinical picture and laboratory evidence of anti-PF4/heparin antibodies. Clinical evaluation may be guided by scoring systems such as the 4Ts and HITT Expert Probability (HEP) score ${ }^{[7]}$. Laboratory tests include immunoassays and functional assays. Immunoassays, such as the PF4/heparin enzymelinked immunosorbent assay (ELISA) or gel assay, are highly sensitive but have limited specificity, while functional tests such as the $14 \mathrm{C}$-serotonin release assay and heparin-induced platelet activation assay are more specific, but are technically challenging ${ }^{[12-14]}$. Relying on immunoassays alone leads to over-diagnosis of HITT, as most patients who form anti-PF4/heparin antibodies do not develop HITT ${ }^{[7]}$. Therefore, clinical probability assessment integrated with immunoassays testing is needed for accurate diagnosis of $\mathrm{HIT}^{[13]}$. Confirming the diagnosis necessitates discontinuation of heparin and initiation of an alternative non-heparin anticoagulant to reduce the risk of thrombosis ${ }^{[12]}$. Commonly used agents include argatroban and fondaparinux. Published evidence supporting the use of direct oral anticoagulants for management of HITT is still limited to case reports ${ }^{[15]}$.

In this present case, the platelet count drop on Day 10 was initially attributed to refractory TTP. This was presumed after the initial exclusion of other common cause of thrombocytopenia such as DIC. Confirmation bias, a cognitive bias that causes physicians to stop searching for additional possibilities beyond the initial preferred hypothesis, may have contributed to delayed suspicion of HITT in this case ${ }^{[16]}$. Theoretically, these types of errors may be mitigated using system-level changes, such as decision support systems. However, a computerized clinical decision support system developed by Riggio and colleagues failed to improve the ability to detect and respond to potential HITT ${ }^{[17]}$.

The outcome of TTP and HITT in our patient was tragic. Although thromboticthrombocytopenicpurpura is well recognized to result in multiorgan dysfunction secondary to formation of micro thrombi, catastrophic presentations of TTP may rarely involve large vessel infarction ${ }^{[6]}$. In our case; the pulmonary embolism and the history of previous exposure to heparin prompted us to investigate for HITT. Furthermore, CAPS was considered but the absence of previous history of antiphospholipid syndrome diagnosis and absence of persistent clinically significant APL positivity make the diagnosis less likely.

This case illustrates the challenges that may arise during treatment of patients with TTP. Definite diagnostic tests for both TTP and HITT (ADAMTS13 level and serotonin release assay, respectively) are only available to a limited number of centers, but a high index of suspicion is required to initiate appropriate therapy.

\section{Conflict of Interest}

The authors have no conflict of interest.

\section{Disclosure}

The authors did not receive any type of commercial support either in forms of compensation or financial for this study. The authors have no financial interest in any of the products or devices, or drugs mentioned in this article.

\section{Ethical Approval}

Obtained.

\section{References}

[1] Scully M. Trends in the diagnosis and management of TTP: European perspective. Transfus Apher Sci 2014; 51(1): 1114.

[2] Schwartz J, Padmanabhan A, Aqui N, Balogun R A, ConnellySmith L, Delaney M. Guidelines on the use of Therapeutic Aphaeresis in Clinical Practice-Evidence-Based Approach from the Writing Committee of the American Society for Aphaeresis: The Seventh Special Issue. J Clin Apheresis 2016; 31(3): 149-162.

[3] Chaturvedi S, Carcioppolo D, Zhang L, McCrae KR. Management and outcomes for patients with TTP: analysis of 100 cases at a single institution. Am J Hematol 2013; 88(7): 560-565.

[4] Thoennissen NH, Müller-Tidow C, Baumann C, Kelsch R, Albrecht O, Sibrowski W Berdel WE, Mesters R. Heparininduced thrombocytopenia vs. plasmapheresis-induced platelet loss in a case of thrombotic thrombocytopenic 
purpura. Transfus Med Hemother 2007; 34(1): 74-77.

[5] Benke S, Moltzan C. Co-existence of heparin-induced thrombocytopenia and thrombotic thrombocytopenic purpura in a postoperative cardiac surgery patient. Am J Hematol 2005; 80(4): 288-291.

[6] Nejat RA, Sugarman R, Schneidmesser E, Katona KC. A catastrophic presentation of thrombotic thrombocytopenic turpura (TTP): large vessel infarction of the right middle cerebral artery. Society of Hospital Medicine 2015. Abstract number: 638. <http://www.shmabstracts.com/ abstract/a-catastrophic-presentation-of-thromboticthrombocytopenic-purpura-ttp-large-vessel-infarction-ofthe-right-middle-cerebral-artery/>.

[7] Coppo P, Veyradier A. Current management and therapeutical perspectives in thrombotic thrombocytopenic purpura. Presse Med 2012; 41(3 Pt 2): e163-176.

[8] Blank M, Asherson RA, Cervera R, Shoenfeld Y. Antiphospholipid syndrome infectious origin. J Clin Immunol 2004; 24(1): 12-23.

[9] Erkan D, Espinosa G, Cervera R. Catastrophic antiphospholipid syndrome: updated diagnostic algorithms. Autoimmun Rev 2010; 10(2): 74-79.

[10] Aguiar CL, Erkan D. Catastrophic antiphospholipid syndrome: how to diagnose a rare but highly fatal disease. Ther Adv Musculoskel Dis 2013; 5(6): 305-314.

[11] Warkentin TE. Heparin-induced thrombocytopenia. Curr Opin Crit Care 2015; 21(6): 576-585.

[12] McKenzie SE, Sachais BS. Advances in the pathophysiology and treatment of heparin-induced thrombocytopenia. Curr Opin Hematol 2014; 21(5): 380-387.

[13] Nagler M, Bachmann LM, ten Cate $H$, ten Cate-Hoek A. Diagnostic value of immunoassays for heparin-induced thrombocytopenia: a systematic review and meta-analysis. Blood 2016; 127(5): 546-557.

[14] Cuker A. Clinical and laboratory diagnosis of heparininduced thrombocytopenia: an integrated approach. Semin Thromb Hemost 2014; 40(1): 106-114.

[15] Miyares MA, Davis KA. Direct-acting oral anticoagulants as emerging treatment options for heparin-induced thrombocytopenia. Ann Pharmacother 2015; 49(6): 735739.

[16] Graber M, Gordon R, Franklin N. Reducing diagnostic errors in medicine: what's the goal? Acad Med 2002; 77(10): 981-992.

[17] Riggio JM, Cooper MK, Leiby BE, Walenga JM, Merli GJ, Gottlieb JE. Effectiveness of a clinical decision support system to identify heparin induced thrombocytopenia. J Thromb Thrombolysis 2009; 28(2): 124-131. 


\title{
فرفرية نقص الصفيحات الخثارية متبوعة بنقص الصفيحات المحفز بالهيبارين
}

\author{
جليلة فيصل زاهر، ومها عبد الرزاق بدوي

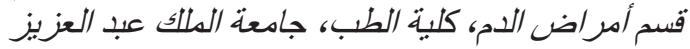 \\ جلة ـ المدلكة العربية السعودية الطبة
}

المستخطص. تحسن مآل المرضى المصابين بفرفرية نقص الصفيحات الخنارية بشكل كبير منذ بدء استخدام فصادة البلازما

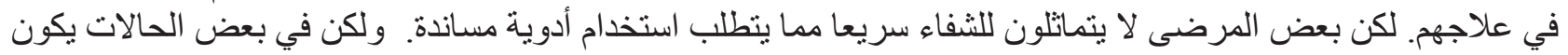

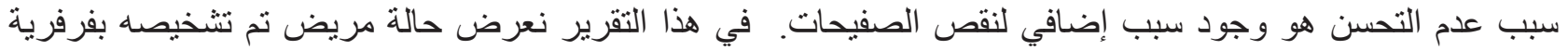

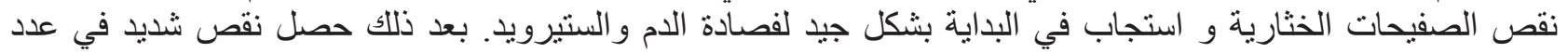

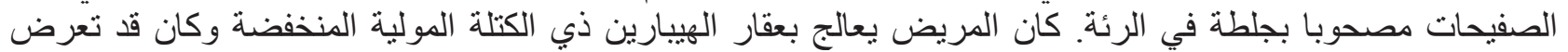

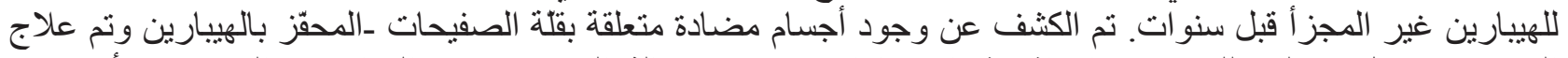

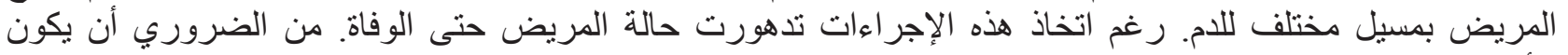

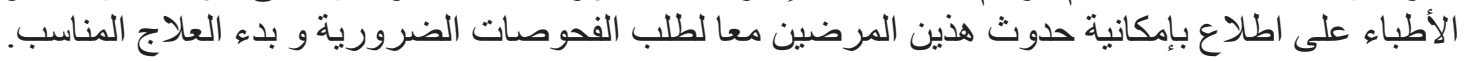

